\title{
Estimating zona pellucida hardness under microinjection to assess oocyte/embryo quality: Analytical and experimental studies
}

\author{
Morteza Khalilian ${ }^{1,2}$, Mojtaba Rezazadeh Valojerdi ${ }^{3,4^{*}}$, Mehdi Navidbakhsh ${ }^{1^{*}}$, Mahmoud Chizari ${ }^{5}$, \\ Poopak Eftekhari-Yazdi ${ }^{4}$ \\ ${ }^{1}$ Mechanical Engineering Department, Iran University of Science and Technology, Tehran, Iran \\ ${ }^{2}$ Mechanical Engineering Department, Urmia University, Urmia, Iran \\ ${ }^{3}$ Department of Anatomy, Faculty of Medical sciences, Tarbiat Modares University, Tehran, Iran \\ ${ }^{4}$ Department of Embryology, Reproductive Biomedicine Research Center, Royan Institute for Reproductive Biomedicine, \\ Academic Center for Education, Culture and Research (ACECR), Tehran, Iran \\ ${ }^{5}$ School of Design and Engineering, Brunel University West London, London, UK

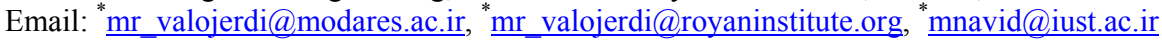

Received 14 March 2013; revised 22 April 2013; accepted 16 May 2013

Copyright (C) 2013 Morteza Khalilian et al. This is an open access article distributed under the Creative Commons Attribution License, which permits unrestricted use, distribution, and reproduction in any medium, provided the original work is properly cited.

\begin{abstract}
The precise determination of zona pellucida (ZP) hardness is largely unknown due to the lack of appropriate measuring and modelling methods. In this study, we have used experimental and theoretical models to describe the mechanical behavior of a single oocyte cell to improve the assisted reproductive technology (ART) outcomes by assessing oocyte/embryo quality. This paper presents the development of: i) a microinjection model to estimate the force of $\mathrm{ZP}$ penetration, ii) a micropipette aspiration model to determine the corresponding hardness, and iii) an experimental procedure to generate the required data for these two models. Our results show that the estimated penetration force provides a performance target for the penetration process during intracytoplasmic sperm injection (ICSI), while the estimated corresponding hardness serves as an indicator of the extent of deformation sustained by the oocyte prior to penetration. Evaluation of these results shows that a routine assessment of ZP hardness under microinjection would allow for the identification of certain oocyte pools for which further manipulation is recommended in order to improve injection, hatching and finally ART outcomes.
\end{abstract}

Keywords: Human Oocyte; Human Embryo; Intracytoplasmic Sperm Injection; Zona Pellucida Hardness

"Corresponding author.

\section{INTRODUCTION}

Single cell manipulation is a widespread process in the field of cellular biology. This process has played key roles in assisted reproductive technology (ART) and other biomedical areas. Over the past decade in vitro fertilization (IVF) was the first assisted reproductive technique offered to patients suffering from reproductive difficulties. In this technique, the oocyte's extracellular layer of glycoprotein, or zona pellucida (ZP), served as a major barrier to sperm-oocyte interaction. Therefore, an alternative to the IVF technique has been developed.

Lanzendorf et al. (1988) first introduced the technique of intracytoplasmic sperm injection (ICSI) and observed normal fertilization following the injection of a single spermatozoon into a human oocyte's cytoplasm [1]. Following this attempt, four years later, Palermo et al. (1992) first reported human pregnancies after ICSI [2]. Since then, ICSI has progressively replaced the previous techniques of gamete micromanipulation as the treatment of choice in cases of male factor infertility and those of failed conventional IVF cycles [3]. Given the widespread use of ICSI, any modifications that can improve its clinical outcomes could possibly benefit a large segment of the patient population.

Although the efficiency of ICSI has been clearly demonstrated $[2,4,5]$, this procedure is typically associated with the degeneration of a significant percentage of treated oocytes [2,3,6,7]. The main cause of this degeneration remains unclear. Some researchers, such as Dumoulin et al. (2001) and Ebner et al. (2003) have speculated that severe mechanical stress during the unusual injection 
procedure may be responsible for oocyte degeneration $[8,9]$. However, on certain occasions, some oocytes show deviations from the presumed optimal ICSI due to a possible impairment in the elasticity of the ZP, which would normally allow the formation of a distinct funnel prior to puncture $[3,6]$. This is characterized by either sudden breakage or delayed penetration of the zona and is likely to result in increased gamete damage $[3,6,8,9]$.

On the other hand, with IVF/ICSI treatment, a high proportion of morphologically normal blastocysts have hatching difficulties [10] due to failure of the embryonic ZP puncture following blastocyst expansion [11]. Several investigators believe that failure to hatch is due mainly to abnormal changes in ZP quality, such as thickening or hardening [12-14].

In order to avoid these inadvertent consequences, several assisted ICSI/hatching techniques such as Piezoassisted ICSI (penetration of the ZP alone with a piezopulse to perform ICSI) $[15,16]$ and laser-assisted ICSI (drilling a microhole through the ZP or thinning the ZP with a laser beam immediately prior to ICSI) [17-20] have been suggested.

Although routine or universal performance of assisted techniques on all oocytes/embryos in IVF/ICSI patients is neither scientific nor appropriate, there is convincing evidence in the literature that assisted techniques increase the survival/implantation capability of some oocytes/ embryos.

Recently, considerable biomedical attention has concentrated on determining the mechanical properties of oocytes as single cells. Numerous studies concerning the mechanical properties of oocytes have been reported [2127]. These studies have shown that the variations within the oocytes' hardness are strongly influenced by mechanical characteristics of the biomembranes (i.e. ZP layer) which isolate them from their immediate surroundings. Therefore, the estimation of zona hardness can be of benefit to quantitative quality assessment because a significant relationship between zona elasticity changes and oocyte/embryo quality has been demonstrated. In this study, we extended the basic model of Wan et al. (2002) to measure the forces required to penetrate the ZP layer in order to study oocytes' structural condition at different breakage modes of the ZP [28]. Also, to model the corresponding Young's modulus, we have proposed a new approach to estimate the hardness of the ZP layer which has utilized the membrane theory.

The present work is in line with our previous researches concerning engineering analysis to study the mechanical properties of an oocyte ZP [26,27] and has been setup to define a novel criterion for predicting the hardness of oocytes and embryos ZP under microinjection.

\section{MATERIAL AND METHOD}

The human oocytes/embryos were donated by infertile couples who did not wish to cryopreserve surplus oocytes/embryos for future replacement. All experiments were done at Royan Institute labs with the permission of the Royan Ethics Committee in collaboration with Iran University of Science and Technology.

\subsection{Preparation of Human Oocytes/Embryos}

Female partners were first superovulated following downregulation, as described elsewhere [26,27,29]. In brief, suppression of pituitary gonadotrophin secretion with the gonadotrophin-releasing hormone agonist $(\mathrm{GnRHa})$ buserelin acetate (Superfact, Hoechst AG, Germany) was commenced during the mid-luteal phase of the preceding ovarian cycle (day 21). Once ovarian suppression was confirmed (serum estradiol $\leq 50 \mathrm{pg} / \mathrm{ml}, \mathrm{LH} \leq 5 \mathrm{IU} / \mathrm{l}$ ), ovarian stimulation was initiated using a subcutaneous injection of $150 \mathrm{IU} /$ day purified human menopausal gonadotrophin (HMG; Pergonal 500, Serono, Italy).

When at least three follicles reached $18 \mathrm{~mm}$ in diameter, GnRHa and HMG were discontinued, and 10,000 IU of human chorionic gonadotrophin (HCG; Organon, Netherlands) was administered. Oocyte retrieval was performed by ultrasound-guided follicle aspiration, 36 - 38 hours after HCG administration. In order to remove the cumulus mass surrounding the oocyte; the obtained cumulus-oocyte complexes were first denuded by using Synvitro $^{\circledR}$ Cumulase $^{\circledR}$ (MediCult, Denmark) and gentle pipetting. The denuded oocytes underwent ICSI and were cultured in G-1 ${ }^{\mathrm{TM}}$ ver 3 (Vitrolife, Kungsbacka, Sweden) supplemented with $10 \%$ recombinant human serum albumin (rHA; Vitrolife) for 24 hours.

\subsection{Experimental Procedures}

A micromanipulation system was used in order to generate empirical data to determine Young's modulus and the penetration force within the deformed membrane of the human oocyte. Both the experimental setup and equipment used for this experiment have been previously reported $[26,27]$. Since measurement of the ZP elastic modulus was essential to determine the penetration force of each oocyte ZP, an aspiration test was initially done. The oocyte was held using a suction pipette and then turned with the aid of a pipette until the polar body came to rest either above (12 o'clock) or below (6 o'clock), Figure 1. Both the pipette and oocyte were sharply focused. The generation of a controlled suction pressure in the pipette enabled the surface of the $\mathrm{ZP}$ to be aspirated into the pipette, which allowed the formation of a hemispherical projection within the pipette. The resulting deformation was recorded using video microscopy and subsequent geometric parameters were measured. Information re- 
garding both the suction pressure and geometric parameters of each oocyte was used to calculate its corresponding elastic modulus.

Applied forces during a microinjection can be estimated from a vision-based biomembrane force sensing. The motion of the injector tip was set towards the center of the cell under the microscope. The oocyte was secured on the holding pipette and the injection pipette was introduced through the zona at the 3 o'clock position (where the injection needle was projected to penetrate into the oocyte) until breakage. For each oocyte, the resulting deformation and type of ZP breakage during the injection process was video recorded.

\subsection{Mechanical Modelling of Oocyte ZP Deformation}

The importance of the mechanical behavior of membrane deformation has been recognized in many research areas such as colloidal science and its biomedical applications have been extensively studied [30-36]. On the experimental front, the elastic properties of a cell wall were quantified by: i) squashing a single cell [37,38]; ii) central point loading membrane clamped between two fixtures [39]; and iii) indenting a capsule by an atomic force microscope (AFM) tip [40-42]. Theoretically, the classical theory of large elastic deformation of a membrane has been determined for: i) gas-filled spherical membranes under plane stress [43]; ii) fluid-filled spherical shells under plane stress [37,42]; iii) fluid-filled spherical shells under pure bending [41]; and iv) homogeneous solid spheres [44].

Despite the above mentioned developments, scant attention has been paid to the mechanical modelling of biological cells, with the exception of Sun's biomembrane point-load model [21]. Wan et al. (2002) [28] developed a theoretical model for the mechanical response of a

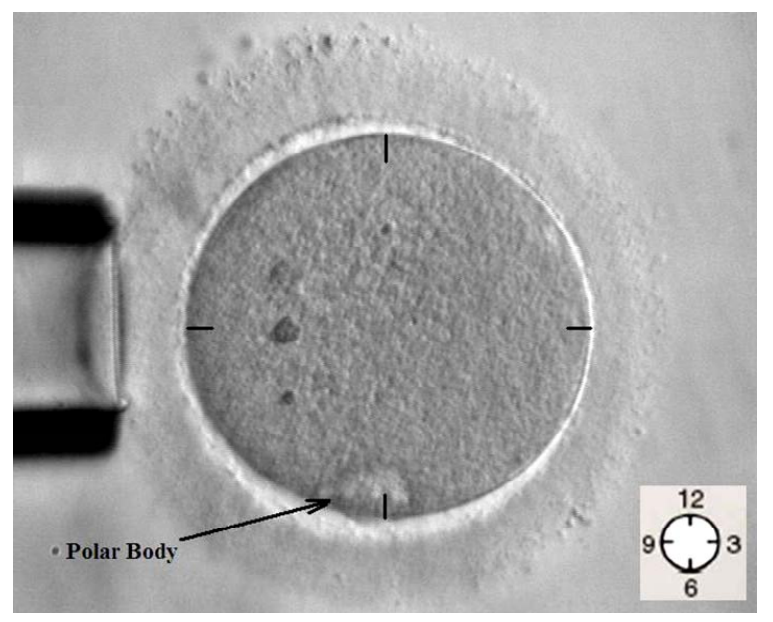

Figure 1. Under microscopic control, a suction pipette supported the oocyte in the different position of the polar body. thin-walled bio-mimetic microcapsule upon indentation by an AFM tip, while Sun et al. (2003) [21] used this model to study the average stress and strain in a mouse ovum ZP. To date, little research has been done that utilized the membrane theory to model biological cells in both microinjection and micropipette aspiration.

\subsubsection{Microinjection Model}

In order to better understand the mechanical responses of oocytes during microinjection, the basic model of Wan et al. (2002) was utilized [28]. Cell wall deformation was considered to be governed by both the bending moment and stretching stress in the presence of a tensile prestress. In Wan's study, it was expressed that creating an indentation dimple by a central load via the AFM tip, as governed by:

$$
k \Delta^{2} w-\sigma h \Delta w=-p+F \delta(r)
$$

with $k=E h^{3} / 12\left(1-v^{2}\right)$ the flexural rigidity; $E$ the elastic modulus; $h$ the film thickness; $v$ the Poisson ratio; $\sigma$ the apparent membrane stress within the dimple; $F$ the total external force exerted by the needle; and $\delta(r)$ the Dirac delta function. The dimple with a radius of $a$, and a depth of $w_{0}$ was created by a needle with a radius of $c$, Figure 2.

Wan et al. (2002) derived an analytical solution for Equation (1) [28]. Thereby, the applied force is given by:

$$
F=\frac{2 \pi E h w_{0}^{3}}{a^{2}\left(1-v^{2}\right)}\left[\frac{3-4 \zeta^{2}+\zeta^{4}+2 \log \zeta^{2}}{\left(1-\zeta^{2}\right)\left(1-\zeta^{2}+\log \zeta^{2}\right)^{3}}\right], \quad \xi=\frac{c}{a}
$$

Due to the similarities between the microinjection process and the AFM indenting manner, we have used Wan's model to analyze the ICSI process.

\subsubsection{Micropipette Aspiration Model}

The micropipette aspiration (MA) technique can be used to quantify the elastic modulus of a cell membrane by pulling a portion of it into a micropipette: when a pressure difference is applied, the membrane is put under tension and sucked inside the pipette [45-47]. Here we

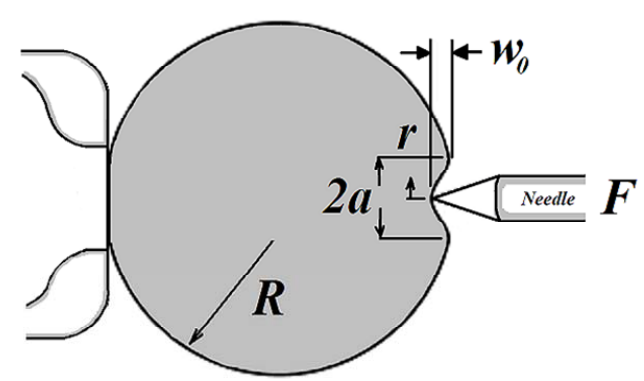

Figure 2. Schematic of a deformed cell during the micro-injection process. 
have applied the MA technique to quantify the elasticity of the ovum's ZP layer under continual aspiration, in combination with the proper mechanical model. Theoretically, the liquid-filled spherical shell theory is used for discussing ZP Young's modulus, which assumes the wall as a thin shell that can be replaced by its median surface. Also throughout modeling, the following are assumed:

1) The ZP encapsulates liquid (cytoplasm) that exerts a uniform hydrostatic pressure on the ZP.

2) The oocyte volume remains constant during the time of the experiment and ZP permeability could be ignored on this time scale.

3) The ZP has a negligible flexural rigidity so that deformation is solely caused by ZP stretching.

4) After aspiration, the oocyte recovers its initial shape and no residual deformation is measured. This suggests that the ZP has a purely elastic behavior and may be regarded as an isotropic elastic shell.

5) The oocyte is free of initial membrane stress or residual stress.

While the movement of ZP projected in the pipette is the result of both an increase in ZP surface area and a decrease in cell volume, experimentation demonstrates that the cell volume may be taken as a constant where small changes in the surface area $\Delta A$ are related to movement of the lead edge of the membrane projection $L$, Figure 3. The area compressibility modulus $k$ can be directly obtained by the relationship:

$$
T=k \cdot \frac{\Delta A}{A_{0}}=k \cdot \alpha
$$

where $\alpha=\Delta A / A_{0}$ denotes the fractional change in area. Therefore the ZP central plane area increment is $\alpha=(H / h)-1$ and $\mathrm{k}$ is the area compressibility modulus of the ZP central plane. The relationship between the pressure difference $\Delta P$ and the imposed membrane tension $T$ directly derives from Laplace's law:

Pressure difference $\Delta \mathrm{P}$ is, therefore, given by:

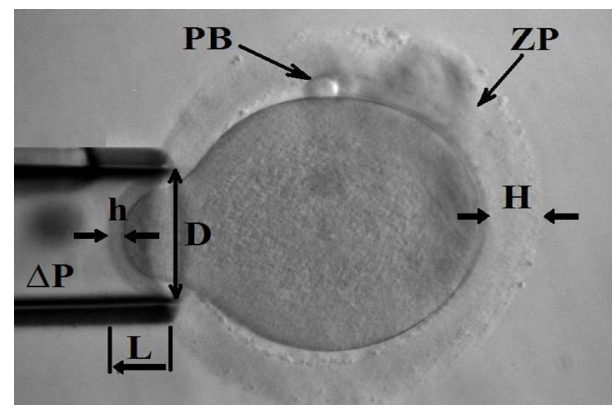

Figure 3. Continuous aspiration of the oocyte into a micropipette; ZP: Zona pellucida; PB: Polar body; $H$ : ZP thickness, $h$ : Thickness of the ZP inside the micropipette; $D$ : Inner diameter of the micropipette; $L$ : Aspiration length of the ZP; $\Delta P$ : Applied negative pressure.

$$
\begin{aligned}
& \Delta P=\frac{2 T}{r}=\frac{2 k \alpha}{r} \\
& \frac{4 k(H-h)}{h(D-h)}=\Delta P
\end{aligned}
$$

$k$ and the elasticity coefficient $E$ relations may infer as follows by the elastic solid physics equation:

$$
\begin{gathered}
\varepsilon_{x}=\frac{1}{E}\left[\sigma_{x}-v\left(\sigma_{y}+\sigma_{z}\right)\right] \\
\varepsilon_{y}=\frac{1}{E}\left[\sigma_{y}-v\left(\sigma_{z}+\sigma_{x}\right)\right] \\
\varepsilon_{z}=\frac{1}{E}\left[\sigma_{z}-v\left(\sigma_{x}+\sigma_{y}\right)\right]
\end{gathered}
$$

In the equation $v=0.5, z$ takes in the ZP radial direction, $x, y$ in tangential. According to the thin spherical shell theory, approximately in the tube it is assumed the ZP two side's intensities of pressure are equal so the internal stress and the strain are even. The boundary conditions are:

$$
\sigma_{z}=0, \varepsilon_{x}=\varepsilon_{y}, \sigma_{x}=\sigma_{y} .
$$

Substitution solid-state physics equation:

$$
\alpha=\frac{1}{E}(1-v) \sigma_{x}=\frac{\sigma_{x}}{2 E},
$$

By:

$$
\alpha=\varepsilon_{x}+\varepsilon_{y}+\varepsilon_{x} \varepsilon_{y}=\frac{\sigma_{x}}{E}+\frac{\sigma_{x}^{2}}{4 E^{2}}
$$

Extracts that:

$$
\sigma_{x}=2 E(\sqrt{1+\alpha}-1)=2 E\left(\sqrt{\frac{H}{h}}-1\right)
$$

ZP central plane tensile force:

$$
F=h \sigma_{x}=k \alpha
$$

Then, it obtains that:

$$
k=\frac{2 h E(\sqrt{H h}-h)}{H-h}
$$

The ZP Young's modulus is found by substituting Equation (12) into Equation (5):

$$
E=\frac{(D-h) \Delta P}{8(\sqrt{H h}-h)}
$$

\section{RESULT}

According to our model, the measured pressure and captured geometries resulting from cellular deformation during the experiment are essential inputs for identifying both the hardness and penetration force of the ZP layer. 
For the former, necessary data are $D, H$ and $h$ which can be directly obtained from the images. For the latter, the data needed would be geometric parameters $a, R$, and $w_{0}$; which are directly obtained from the captured images in Figure 4.

A method of edge detection was used to estimate these parameters based on the differences in pixel intensity between the curve of the deformed membrane and its background, Figure 5(a). A set of seven images which corresponded to the seven values of indentation depth, were used to generate the values of applied forces that ranged from initial contact to the point just prior to pene- tration of the ZP, Figure 5(b). With $c=7 \mu \mathrm{m}$ and $h=$ $20.3 \mu \mathrm{m}$, Equation (3) yields the seven points plotted in Figure 5(c) which show the values of the needle's applied forces that correspond to the seven values of indentation depth. Figure 5(c) shows the typical force and deformation measurement process on a human oocyte ZP. As shown in this Figure, forces increase nonlinearly as deformation increases. When deformation reaches approximately $100 \mu \mathrm{m}$, the ZP layer is punctured, which results in the maximum forces measured, i.e., the puncturing forces. The puncturing force is approximately 90 $\mu \mathrm{N}$.

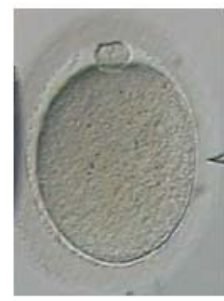

1

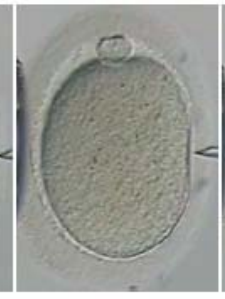

2

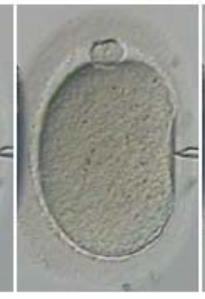

3

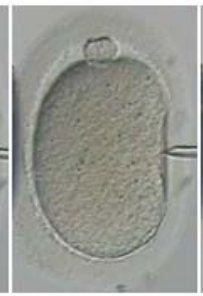

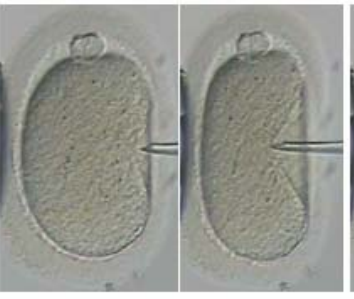

5

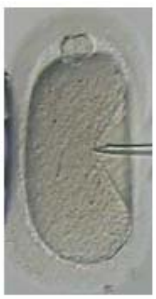

7

Figure 4. The seven stages of a human oocyte ZP indentation caused by a needle.
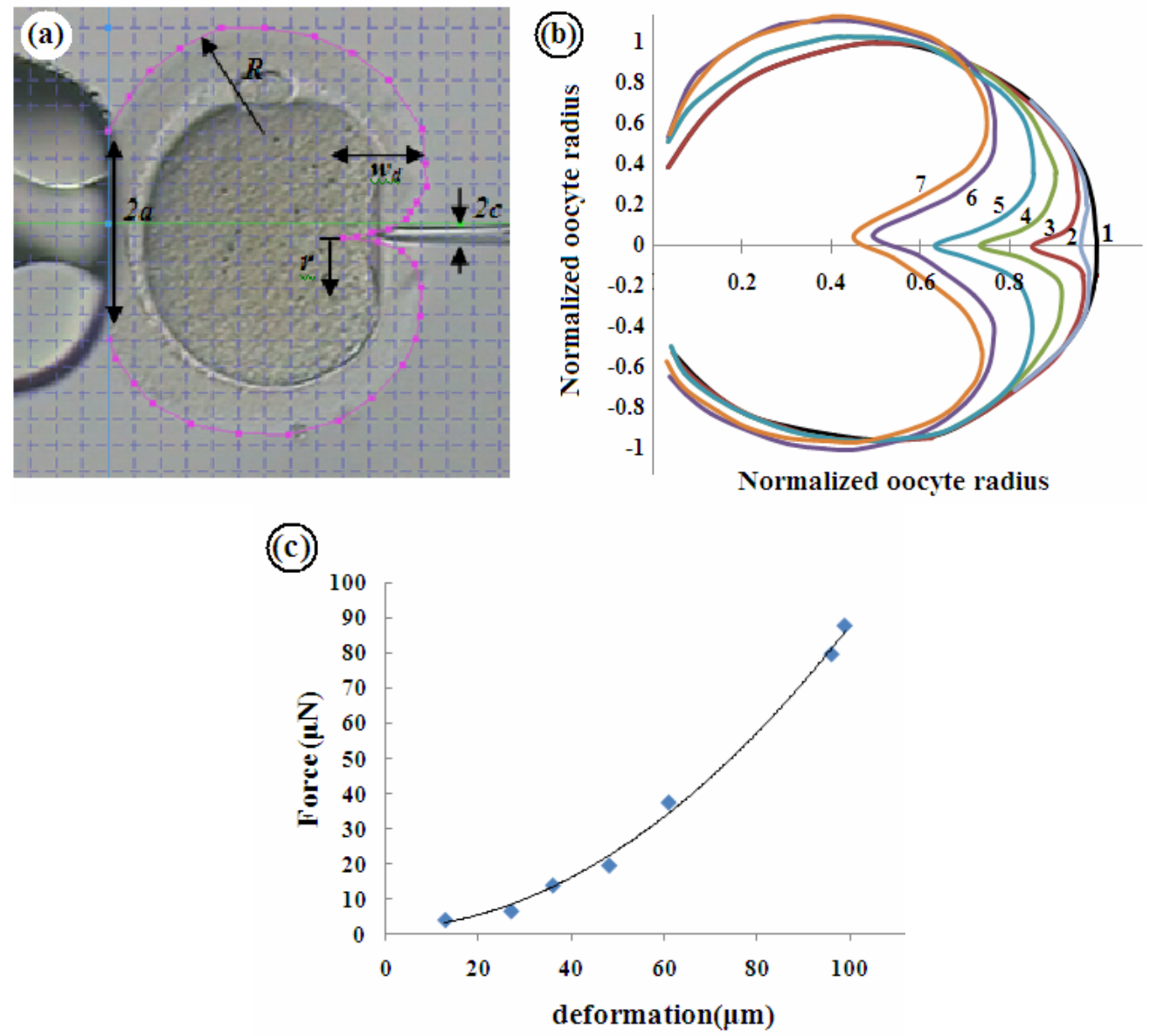

Figure 5. (a) Sketch of a deformed oocyte under the force of a needle, (b) Tracking the pattern of the deformed oocyte ZP, (c) Force-deformation curve of a human oocyte ZP. 
Figure 6 compares the penetration forces of three different oocyte ZPs obtained from the same patient. Details of the experiments have been reported in Table 1.

The ZP was punctured by following the penetration of the injection pipette into the oocyte. Failure modes can be classified into four types: Type A, in which the ZP punctures with slight membranous stretching immediately upon insertion of the pipette into the oocyte; Type

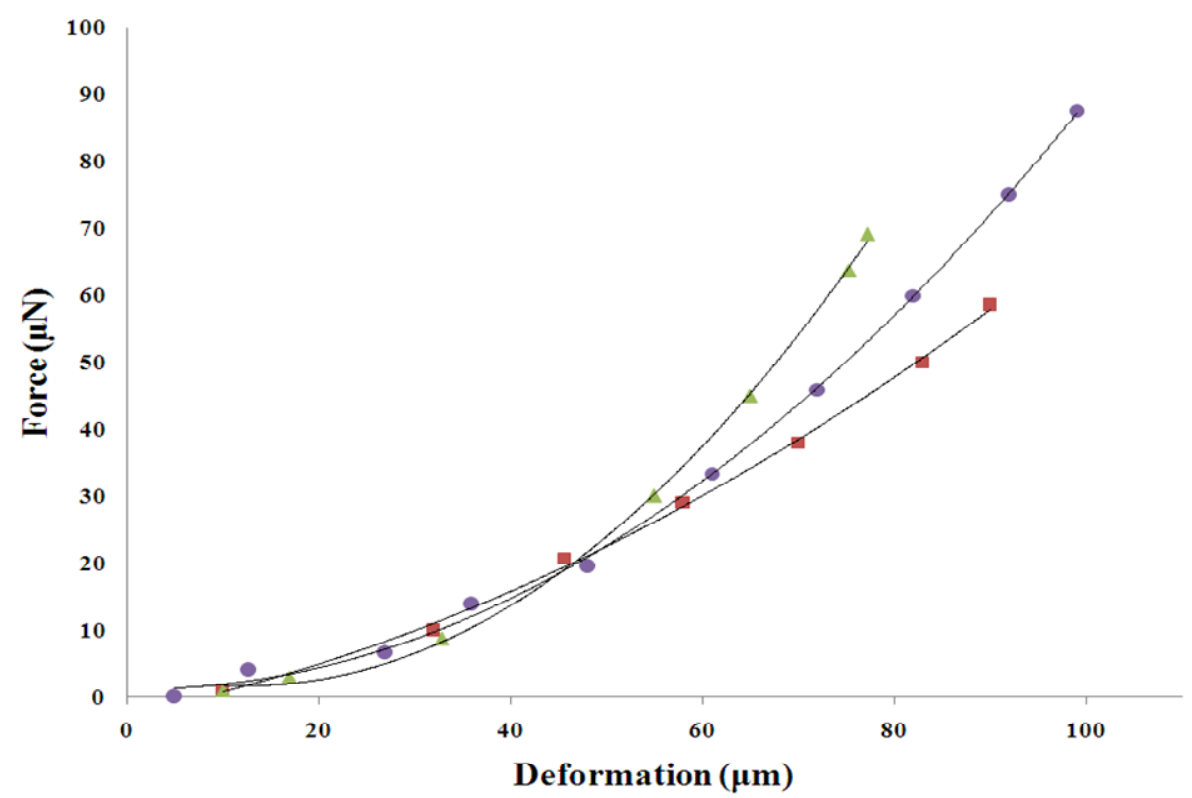

Figure 6. ZP failure mode. A: Puncture of an oocyte ZP occurring just after insertion of the needle, B: Puncture of an oocyte ZP when the needle penetrates into a depth of $1 / 3$ of the oocyte's diameter, C: Puncture of an oocyte ZP when the needle penetrates to the center of the oocyte, D: Puncture of an oocyte ZP when the needle penetrates into 3/4 of the oocyte's diameter.

Table 1. Typical human oocyte characteristics. $d_{\text {cell }}$ : Oocyte diameter; $H_{\mathrm{ZP}}$ : ZP thickness; $d$ : Needle displacement; $E$ : ZP ela- sticity; $F$ : Penetration force.

\begin{tabular}{|c|c|c|c|}
\hline Human Oocyte & Oocyte 1 & Oocyte 2 & Oocyte 3 \\
\hline$d_{\text {cell }}(\mu \mathrm{m})$ & 171 & 181 & 179 \\
\hline$H_{\mathrm{ZP}}(\mu \mathrm{m})$ & 15.1 & 17.5 & 20.3 \\
\hline$d(\mu \mathrm{m})$ & $77<d_{\text {cell }} / 2$ & $90 \approx d_{\mathrm{cell}} / 2$ & $99>d_{\text {cell }} / 2$ \\
\hline$E(k P a)$ & 4.22 & 4.02 & 3.19 \\
\hline$F(\mu \mathbf{N})$ & 69.23 & 58.6 & 87.6 \\
\hline Breakage mode & Sudden & Normal & Difficult \\
\hline $\begin{array}{c}\text { Prior to } \\
\text { needle insertion }\end{array}$ & & & \\
\hline $\begin{array}{c}\text { Prior to } \\
\text { ZP Puncture }\end{array}$ & & & \\
\hline
\end{tabular}



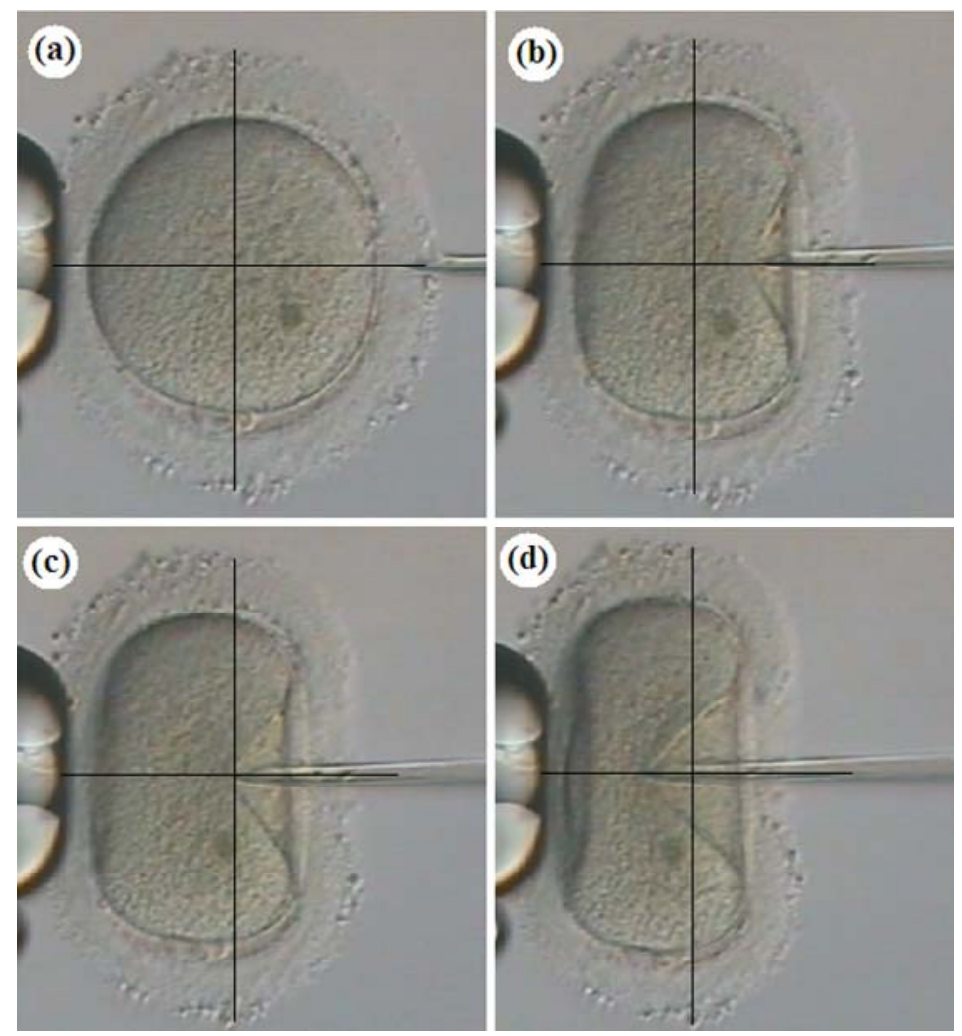

Figure 7. Force-deformation curves of a typical human oocyte ZP. Filled triangle, oocyte 1 ; filled square, oocyte 2 ; filled cir- cle, oocyte 3 .

$\mathrm{B}$, where the ZP punctures suddenly when the pipette penetrates approximately one-third of its diameter into the oocyte; Type $\mathrm{C}$, in which the ZP punctures normally when the pipette reaches the center; and Type $\mathrm{D}$, it is difficult to puncture $\mathrm{ZP}$ when the pipette is inserted to threefourths of its diameter, Figure 7. The failure modes were observed during ICSI by individuals who monitored the procedure.

\section{DISCUSSION}

The objective of present study was to use an appropriate method to measure the hardness of the ovum's extracellular envelope (ZP) and to define a novel criterion for predicting the quality of oocytes and embryos under microinjection. In this regard, we have used suitable mathematical models to calculate the elastic properties of the ZP. Our findings support the hypothesis that mechanical properties of certain types of cells may potentially be used to quantitatively reflect the state of their structural components and health $[34,48]$.

In ICSI procedure treated oocytes may, in part, be identified by their morphological alterations [9]. The main objective of ICSI is to pierce a needle holding the sperm through the ZP and oolemma, and release the sperm in the deep area of the ooplasm.

In our study, some of the oocytes showed deviations from a presumed optimal ICSI. The elasticity of the outer structures, which would normally allow the formation of a distinct funnel prior to puncture, appeared somewhat impaired in those oocytes. This was characterized by either sudden breakage or delayed penetration of zona, Figure 6, and likely resulted in increased damage of the oocytes $[3,6,8]$.

In this study, we attempted to analytically evaluate and compare the human oocyte ZP hardness with the estimated corresponding puncture force, Table 1. It was concluded that high forces (about 1.2 to 1.5 times) were needed for both abnormal puncture patterns when compared to the normal mode, Figure 7. Therefore, both sudden and difficult puncture patterns may serve as an impaired ZP indication.

Degeneration of oocytes occurs with a resistant ZP (when the oocyte has to be strongly compressed in order to be penetrated by the ICSI needle) during ICSI [6]. In such cases a higher pressure must be applied to pass through all anatomical structures, possibly causing mechanical damage to the meiotic spindle and cytoskeleton [9]. In order to prevent this possible dilemma, specific methods of microinjection that avoid or minimize the chance of degeneration are needed. There is evidence that assisted hatching may be applied in embryos derived from oocytes with difficult penetration of the ZP; in or- 
der to help the embryos escape from their hard ZP and attach to the womb $[9,18]$.

Previous retrospective studies have reported that embryos resulting from cycles with fragile zona oocytes exhibit a higher percentage of fragmentation. This framentation is associated with significantly poorer embryo quality $[3,6,8]$ and a reduced rate of compaction as compared to cycles without fragile ZPs [49]. Consequently, such embryos may not have the ability to implant and are subsequently excluded from the treatment process [19].

According to the experimental examination of this study, four different cases of ZP puncture patterns during the ICSI procedure have been observed. "Type A" with no puncture, "Type B" with a sudden puncture, "Type C" with a normal puncture; and "Type D" with a difficult puncture. The selection of an oocyte cell is very important to obtain satisfactory ICSI results. Thus further manipulation is recommended with oocytes/embryos in ICSI patients.

These findings and the present investigation demonstrate that the success rates of ICSI technique and assisted ICSI/hatching methods are largely dependent on the mode by which the ZP is breached during ICSI as well as the size of the created funnel. Therefore, it is suggested that the ZP puncture mode during ICSI may be applied in human ART to assess oocyte/embryo quality to determine selective assisted techniques.

This distinction may have implications for whether a good quality embryo is able to undergo normal zona hatching during implantation or whether a different method of zona assisted hatching is needed.

\section{CONCLUSION}

This study provides a new method for estimating the ZP hardness and quality evaluation of the oocyte/embryo during ICSI procedure and may be used for a better understanding of the key role of oocyte ZP in ART.

\section{ACKNOWLEDGEMENTS}

This work was supported by Royan Institute, Tehran, Iran. We would like to thank the Embryology Department of Royan Institute for their assistance during this research and also the patients who donated their oocytes or embryos. The authors also do not have any financial interest or any other conflict of interests.

\section{REFERENCES}

[1] Lanzendorf, S.E., Maloney, M.K., Veek, L.L., Slusser, J., Hodgen, G.D. and Rosenwaks, Z. (1998) A preclinical evaluation of pronuclear formation by microinjection of human spermatozoa into human oocytes. Fertility and Sterility, 49, 835-842.

[2] Palermo, G., Joris, H., Devroey, P. and Van Steirteghem,
A.C. (1992) Pregnancies after intracytoplasmic injection of single spermatozoon into an oocyte. Lancet, 340, 1718. doi:10.1016/0140-6736(92)92425-F

[3] Palermo, G.D., Alikani, M., Bertoli, M., Colombero, L.T., Moy, F., Cohen, J. and Rosenwaks, Z. (1996) Oolemma characteristics in relation to survival and fertilization patterns of oocytes treated by intracytoplasmic sperm injection. Human Reproduction, 11, 172-176. doi:10.1093/oxfordjournals.humrep.a019012

[4] Van Steirteghem, A.C., Nagy, Z., Joris, H., Liu, J., Staessen, C., Smitz, J., Wisanto, A. and Devroey, P. (1993) High fertilization and implantation rates after intracytoplasmic sperm injection. Human Reproduction, 8, 10611066.

[5] Mansour, R. (1998) Intracytoplasmic sperm injection: A state of the art technique. Human Reproduction Update, $\mathbf{4}$, 43-56. doi:10.1093/humupd/4.1.43

[6] Nagy, Z.P., Liu, J., Joris, H., Bocken, G., Desmet, B., Van Ranst, H., Vankelecom, A., Devroey, P. and Van Steirteghem, A.C. (1995) The influence of the site of sperm deposition and mode of oolemma breakage at intracytoplasmic sperm injection on fertilization and embryo development rates. Human Reproduction, 10, 31713177.

[7] Yavas, Y., Roberge, S., Khamsi, F., Shirazi, P., Endman, M.W. and Wong, J.C. (2001) Performing ICSI using an injection pipette with the smallest possible inner diameter and a long taper increases normal fertilization rate, decreases incidence of degeneration and tripronuclear zygotes, and enhances embryo development. Journal of Assisted Reproduction and Genetics, 18, 426-435. doi:10.1023/A:1016634704469

[8] Dumoulin, J.C.M., Coonen, E., Bras. M., Bergers-Janssen, J.M., Ignoul-Vanvuchelen, R.C., van Wissen, L.C., Geraedts, J.P. and Evers, J.L. (2001) Embryo development and chromosomal anomalies after ICSI: Effect of the injection procedure. Human Reproduction, 16, 306-312. doi:10.1093/humrep/16.2.306

[9] Ebner, T., Moser, M., Sommergruber, M., Puchner, M., Wiesinger, R. and Tews, G. (2003) Developmental competence of oocytes showing increased cytoplasmic viscosity. Human Reproduction, 18, 1294-1298. doi:10.1093/humrep/deg232

[10] Zhang, X., Rutledge, J. and Armstrong, D.T. (1991) Studies on zona hardening in rat oocytes that are matured in vitro in a serum-free medium. Molecular Reproduction and Development, 28, 292-296. doi: $10.1002 / \mathrm{mrd} .1080280312$

[11] Fong, C.Y., Bongso, A., Sathananthan, H., Ho, J. and Ng, S.C. (2001) Ultrastructural observations of enzymatically treated human blastocysts: Zona-free blastocyst transfer and rescue of blastocysts with hatching difficulties. $\mathrm{Hu}$ man Reproduction, 16, 540-546.

doi:10.1093/humrep/16.3.540

[12] Edwards, R.G., Fishel, S.B., Cohen, J., Fehilly, C.B., Purdy, J.M. and Salter, J.M. (1984) Factors influencing the success of in vitro fertilization for alleviating human infertility. Journal in Vitro Fertilization and Embryo Transfer, 1, 3-23. doi:10.1007/BF01129615 
[13] Cohen, J., Alikani, M., Trowbridge, J. and Rosenwacks, Z. (1992) Implantation enhancement by selective assisted hatching using zona drilling of human embryos with poor prognosis. Human Reproduction, 7, 685-691.

[14] Bertrand, E., Van den Bergh, M. and Englert, Y. (1996) Clinical parameters influencing human zona pellucida thickness. Fertility and Sterility, 66, 408-411.

[15] Huang, T., Kimura, Y. and Yanagimachi, R. (1996) The use of piezo micromanipulation for intracytoplasmic sperminjection of human oocytes. Journal of Assisted Reproduction and Genetic, 13, 320-328. doi:10.1007/BF02070146

[16] Yanagida, K., Katayose, H., Yazawa, H., Kimura, Y., Konnai, K. and Sato, A. (1999) The usefulness of a Piezo-micromanipulator in intracytoplasmic sperm injection in humans. Human Reproduction, 14, 448-453. doi:10.1093/humrep/14.2.448

[17] Rienzi, L.B., Greco, E., Ubaldi, F., Iacobelli, M., Martinez, F. and Tesarik, J. (2001) Laser-assisted intracytoplasmic sperm injection. Fertility and Sterility, 76, 10451047. doi:10.1016/S0015-0282(01)02861-8

[18] Nagy, Z.P., Oliveira, S.A., Abdelmassih, V. and Abdelmassih, R. (2002) Novel use of laser to assist ICSI for patients with fragile oocytes: A case report. Reproductive and Biomedicine Online, 4, 27-31. doi:10.1016/S1472-6483(10)61911-6

[19] Abdelmassih, S., Cardoso, J., Abdelmassih, V., Dias, J.A., Abdelmassih, R. and Nagy, Z.P. (2002) Laser-assisted ICSI: A novel approach to obtain higher oocyte survival and embryo quality rates. Human Reproduction, 17, 26942699. doi:10.1093/humrep/17.10.2694

[20] Moser, M., Ebner, T., Sommergruber, M., Gaisswinkler, U., Jesacher, K., Puchner, M., Wiesinger, R. and Tews, G. (2004) Laser-assisted zona pellucida thinning prior to routine ICSI. Human Reproduction, 19, 573-578. doi:10.1093/humrep/deh093

[21] Sun, Y., Wan, K., Roverts, K., Bischof, J. and Nelson, B.J. (2003) Mechanical property characterization of mouse zona pellucida. IEEE Transaction on Nanobioscience, $\mathbf{2}$, 279-286. doi:10.1109/TNB.2003.820273

[22] Murayama, Y., Constantinou, C.E. and Omata, S. (2004) Micro-mechanical sensing platform for the characterization of the elastic properties of the ovum via uniaxial measurement. Journal of Biomechanics, 37, 67-72. doi:10.1016/S0021-9290(03)00242-2

[23] Murayama, Y., Mizuno, J., Kamakura, H., Fueta, Y., Nakamura, H., Akaishi, K, Anzai, K., Watanabe, A., Inui, H. and Omata, S. (2006) Mouse zona pellucida dynamically changes its elasticity during oocyte maturation, fertilization and early embryo development. Human Cell, 19, 119-125. doi:10.1111/j.1749-0774.2006.00019.x

[24] Murayama, Y., Yoshida, M., Mizuno, J., Nakamura, H., Inoue, S., et al. (2008) Elasticity Measurement of Zona Pellucida Using a Micro Tactile Sensor to Evaluate Embryo Quality. Journal of Mammalian Ova Research, 25, 8-16. doi:10.1274/jmor.25.8

[25] Kim, D.H., Hwang, C.N., Sun, Y., Lee, S.H., Kim, B. and Nelson, B.J. (2006) Mechanical analysis of chorion softening in prehatching stages of zebrafish embryos. IEEE
Transaction on Nanobioscience, 5, 89-94. doi:10.1109/TNB.2006.875054

[26] Khalilian, M., Navidbakhsh, M., Rezazadeh-Valojerdi, M., Chizari, M. and Eftekhari-Yazdi, P. (2009) Estimating young's modulus of zona pellucida by micropipette aspiration in combination with theoretical models of ovum. Journal of the Royal Society Interface, 7, 689-694. doi:10.1098/rsif.2009.0380

[27] Khalilian, M., Navidbakhsh, M., Rezazadeh-Valojerdi, M., Chizari, M. and Eftekhari-Yazdi, P. (2010) Alteration in the mechanical properties of human ovum zona pellucida following fertilization: Experimental and analytical studies. Experimental Mechanics, 51, 175-182. doi:10.1007/s11340-010-9357-z

[28] Wan, K.T., Chan, V. and Dillard, D. (2002) Constitutive equation for elastic indentation of a thin-walled bio-mimetic microcapsule by an atomic force microscope tip. Colloids and Surfaces B: Biointerfaces, 27, 241-248. doi:10.1016/S0927-7765(02)00073-5

[29] Porter, R.N., Smith, W., Craft, I.L., Abdulwahid, N.A. and Jacobs, H.S. (1984) Induction of ovulation for in vitro fertilization using buserelin and gonadotropins. Lancet, 2, 1284-1285. doi:10.1016/S0140-6736(84)92840-X

[30] Evans, E.A. and Skalak, R. (1980) Mechanics and thermodynamics of biomembranes. CRC Press Inc., Boca Raton.

[31] Cheng, L.Y. (1987) Deformation analyses in cell and developmental biology. Part I-Format methodology. Journal of Biomechanical Engineering, 109, 10-17. doi:10.1115/1.3138634

[32] Cheng, L.Y. (1980) Deformation analyses in cell a developmental biology. Part II: Mechanical experiments on cell. Journal of Biomechanical Engineering, 109, 18-24. doi: $10.1115 / 1.3138635$

[33] Smith, A.E., Moxham, K.E. and Middelberg, A.P.J. (1998) On uniquely determining cell-wall material properties with the compression experiment. Chemical Engineering Science, 53, 3913-3922. doi:10.1016/S0009-2509(98)00198-5

[34] Zhu, C., Bao, G. and Wang, N. (200) Cell Mechanics: Mechanical response, cell adhesion, and molecular deformation. Annual Review of Biomedical Engineering, 2, 189-226. doi:10.1146/annurev.bioeng.2.1.189

[35] Foo, J.J., Chan, V. and Liu, K.K. (2006) Coupling bending and shear effects on liposome deformation. Journal of Biomechanics, 39, 2338-2343. doi:10.1016/j.jbiomech.2005.07.008

[36] Liu, K.K. (2006) Deformation behaviour of soft particles: A review. Journal of Physics D: Applied Physics, 39, 189-199. doi:10.1088/0022-3727/39/11/R01

[37] Lardner, T.J. and Pujara, P. (1980) Compression of spherical cells. Mechanics Today, 5, 161-176.

[38] Liu, K.K., Williams, D.R. and Briscoe, B.J. (1999) A new method to characterize the mechanical properties of a single cell. Medical and Biological Engineering and Computing, 37, 1506-1507.

[39] Ju, B.F., Liu, K.K., Ling, S.F. and Ng, W.H. (2002) A novel technique for characterizing elastic properties of 
thin biological membrane. Mechanics of Materials, 34, 749-754. doi:10.1016/S0167-6636(02)00182-5

[40] Boulbitch, A. (2000) Deformation of the envelope of a spherical Gram-negative bacterium during the atomic force microscopic measurement. Journal of Electron Microscopy, 49, 459-462.

doi:10.1093/oxfordjournals.jmicro.a023829

[41] Arnoldi, M., Fritz, M., Baeuerlein, E., Radmacher, M., Sackmann, E. and Boulbitch, A. (2000) Bacterial turgor pressure can be measured by atomic force microscopy. Physical Review, 62, 1034-1044.

[42] Yao, X., Walter, S., Burke, S., Stewart, M.H., Jericho, M.H., Pink, D., Hunter, R. and Beveridge, T.J. (2002) Atomic force microscopy and theoretical considerations of surface properties and turgor pressures of bacteria. Colloids and Surfaces B: Biointerfaces, 23, 213-230. doi:10.1016/S0927-7765(01)00249-1

[43] Feng, W.W. and Yang, W.H. (1973) On the contact problem of an inflated spherical nonlinear membrane. Transaction of the American Society of Mechanical Engineers: Journal of Applied Mechanics, 40, 209-214. doi:10.1115/1.3422928

[44] Tatara, Y. (1991) On compression of rubber elastic sphere over a large range of displacements-Part I: Theoretical study. ASME Journal of Engineering Material and Technology, 113, 285-291. doi:10.1115/1.2903407

[45] Evans, E. and Yeung, A. (1973) New membrane concept applied to the analysis of fluid shear- and micropi-pettedeformed red blood cells. Biophysics Journal, 13, 941954. doi:10.1016/S0006-3495(73)86036-9

[46] Evans, E. and Yeung, A. (1989) Apparent viscosity and cortical tension of blood granulocytes determined by micropipette aspiration. Biophysics Journal, 56, 151-160. doi:10.1016/S0006-3495(89)82660-8

[47] Hochmuth, R.M. (2000) Micropipette aspiration of living cells. Journal of Biomechanics, 33, 15-22. doi:10.1016/S0021-9290(99)00175-X

[48] Bao, G. and Suresh, S. (2003) Cell and molecular mechanics of biological materials. Nature Materials, 2, 715725 .

[49] Richter, K.S., Davis, A., Carter, J., Greenhouse, S.J., Mottla, G.L. and Tucker, M.J. (2006) No advantage of laser-assisted over conventional intracytoplasmic sperm injection: A randomized controlled trial. Journal of Experimental \& Clinical Assisted Reproduction, 3, 1-5. doi:10.1186/1743-1050-3-5 